\title{
Reflexões sobre recursos tecnológicos: ajudas técnicas, tecnologia assistiva, tecnologia de assistência e tecnologia de apoio
}

\section{Reflections on technological resources: aids techniques, assistive technology, technology of assistance and technology of support}

\author{
Eucenir Fredini Rocha ${ }^{1}$, Maria do Carmo Castiglioni ${ }^{2}$
}

ROCHA, E. F.; CASTIGLIONI, M. C. Reflexões sobre recursos tecnológicos: ajudas técnicas, tecnologia assistiva, tecnologia de assistência e tecnologia de apoio. Rev. Ter. Ocup. Univ. São Paulo, v. 16, n. 3, p. 97-104, set./dez., 2005.

\begin{abstract}
RESUMO: Os recursos tecnológicos especializados, equipamentos de ajuda, estão sendo utilizados e produzidos com mais freqüência nos últimos anos e se cunhou alguns termos que sintetizam essa produção. Várias são as terminologias utilizadas no Brasil para definir o que são os recursos tecnológicos: Tecnologia Assistiva (EUA), Tecnologia de Assistência (CIF/OMS) e Tecnologia de Apoio (Comissão Européia/ EUSTAT) e Ajudas Técnicas (Ministério da Saúde). Com as diferentes concepções seguem classificações diversas desse campo de conhecimento, bem como formas singulares de entender o tema. As estratégias e procedimentos decorrentes, também são distintos e ulteriores às diferentes compreensões. $\mathrm{O}$ artigo pretende discutir os recursos tecnológicos para além dos aspectos práticos de sua utilização no cotidiano. Parte do suposto de que a TA (Tecnologia Assistiva, de Apoio, de Assistência ou Ajudas Técnicas) é um fenômeno multidimensional, que envolve aspectos mecânicos, biomecânicos, ergonômicos, funcionais, cinesiológicos, éticos, estéticos, políticos, afetivos, subjetivos e como tal deve ser analisado. Apresenta, também, os conceitos que estão implicados, de acordo com as diferentes concepções de reabilitação. E, faz uma análise quanto ao sentido e finalidade desses recursos no processo de inclusão social da pessoa com deficiência.
\end{abstract}

DESCRITORES: Tecnologia biomédica/tendências, Tecnologia/tendências, Equipamentos de auto-ajuda, Pessoas portadoras de deficiência/reabilitação, Reabilitação.

\footnotetext{
1. Profa. Dra. do Departamento de Fisioterapia, Fonoaudiologia e Terapia Ocupacional da FMUSP. eucenir@usp.br

2. Profa. Dra. do Departamento de Fisioterapia, Fonoaudiologia e Terapia Ocupacional da FMUSP. carminha_cas@hotmail.com Endereço para correspondência: Rua Cipotânea, 51. Cidade Universitária. São Paulo, SP. CEP: 05360-160.
} 


\section{Apresentação: relações dos recursos tecnológicos com independência e autonomia}

V

ive-se um momento em que a tecnologia é altamente valorizada. $\mathrm{O}$ mundo atual funciona a partir de tecnologias cada vez mais sofisticadas e a crença na sua eficácia é significativa. Sem dúvida, os recursos técnicos podem facilitar a vida moderna do homem. No entanto, é importante estar atento ao fato de que ela pode não contemplar a totalidade das necessidades das pessoas.

No campo da intervenção em reabilitação de pessoas com deficiências, incapacidades ou idosas, muitas são as expectativas dos profissionais em relação às contribuições que a tecnologia pode trazer para seus usuários a fim de proporcionar independência e autonomia. Gradativamente são feitos investimentos na direção de produzir e aplicar conhecimentos em produtos específicos para essa população, que passaremos a denominar de Recursos Tecnológicos (RT).

Os RTs especializados ou equipamentos de ajuda, estão sendo utilizados e produzidos com mais freqüência nos últimos anos e se cunhou alguns termos que são utilizados ao se referir à essa produção. Várias são as terminologias utilizadas no Brasil para definir o que são os recursos tecnológicos: Tecnologia Assistiva (EUA), Tecnologia de Assistência (CIF/OMS) e Tecnologia de Apoio (Comissão Européia/EUSTAT) e Ajudas Técnicas (Ministério da Saúde).

Com as diferentes concepções seguem classificações diversas desse campo de conhecimento, bem como formas singulares de entender o tema. As estratégias e procedimentos decorrentes, também são distintos e ulteriores às diferentes compreensões.

Pretende-se aqui, analisar criticamente as TAs (Ajudas Técnicas, Tecnologias Assistiva, de Apoio, de Assistência) - que está circunscrita na tecnologia, a qual pode ser entendida como a confluência entre ciência, técnica e interesse econômico. Partimos do suposto de que a TA é um fenômeno multidimensional, que envolve aspectos mecânicos, biomecânicos, ergonômicos, funcionais, cinesiológicos, éticos, estéticos, políticos, afetivos, subjetivos e como tal deve ser analisado.

Nessa breve apresentação, outro aspecto deve ser destacado - a decisão do uso do recurso é da pessoa com deficiência ou idoso - pois isso pertence ao processo de autonomia, onde o significado do uso deve estar contextualizado na vida do usuário.

Pode-se, questionar o que vem a ser independência e autonomia, e, em que medida os recursos tecnológicos contribuem para esse processo junto às pessoas com deficiências ou incapacidades e idosos.

Segundo o Novo Aurélio Século XXI: o dicionário da língua portuguesa (1999)

independência é um estado ou condição de quem ou do que é independente, de quem ou do que tem liberdade ou autonomia. Independente é aquele que está livre de qualquer dependência ou sujeição, que é senhor das próprias decisões com autonomia. Autonomia é a faculdade de se governar por si mesmo, liberdade ou independência moral ou intelectual. Do ponto de vista ético é a propriedade pela qual o homem pretende poder escolher as leis que regem sua conduta. Autonomia significa autodeterminação.

No caso da pessoa com deficiência ou idoso, muitas são as situações em que ela pode ser dependente de terceiros ou de equipamentos de ajuda. E, autonomia que se refere ao ato de governar a si próprio, pode ser mesmo quando se recorre à ajuda de outras pessoas ou de dispositivos tecnológicos?

Se o recurso tecnológico representar um instrumento de emancipação e independência nas suas atividades e de realização pessoal, ele poderá promover a autonomia, mas não o garantindo por si só. Contudo, se a tecnologia representar um fracasso, significar uma acusação de sua impotência, um atributo negativo de denúncia social e psicológica de suas limitações, ele não irá promover independência e autonomia.

Mapeando os conceitos de recursos tecnológicos: ajudas técnicas, tecnologia assistiva, tecnologia de assistência e tecnologia de apoio

Nos EUA, Tecnologia Assistiva foi definida em 1988, através de uma lei pública (Technology-Related Assistance for Individuals with Disabilities Act - Public 100-407), como:

qualquer item, peça de equipamento ou sistema de produtos, quando adquiridos comercialmente, modificados, ou feito sob medida, que é usado para aumentar, manter ou melhorar as habilidades funcionais do indivíduo com limitações funcionais (MELLO, 1997).

Segundo Mello (1997), a tecnologia é considerada Assistiva quando é usada para auxiliar no desempenho funcional de atividades, reduzindo incapacidades para a realização de atividades da vida diária e da vida prática, nos diversos domínios do cotidiano. É diferente da tecnologia reabilitadora, usada, por exemplo, para auxiliar na recuperação de movimentos diminuídos.

A Tecnologia Assistiva envolve tanto o objeto, ou seja, a tecnologia concreta (o equipamento ou instrumento), quanto o conhecimento requerido no processo de avaliação, criação, escolha e prescrição, isto é, a tecnologia 
teórica. Tem como áreas de aplicação: adaptações para atividades da vida diária; sistemas de comunicação alternativa; dispositivos para utilização de computadores; unidades de controle ambiental; adaptações estruturais em ambientes domésticos, profissionais ou público; adequação da postura sentada; adaptações para déficits visuais e auditivos; equipamentos para mobilidade; adaptações em veículos.

A Tecnologia de Assistência da Classificação Internacional de Funcionalidade, Incapacidade e Saúde (CIF/OMS, 2003) são definidos como ...qualquer produto, instrumento, equipamento ou tecnologia adaptado ou especialmente projetado para melhorar a funcionalidade de uma pessoa incapacitada (p. 201).

Em matéria recente, veiculada pela Internet, o tema é abordado pela Comissão Européia - (EUSTAT- Emporwing Users Through_Assistive Technology, 1999) que intenta esclarecer o sentido que dá ao termo adotado como Tecnologia de Apoio.

"Tecnologia" indica objetos físicos (dispositivos ou equipamentos) e também contextos organizacionais ou "modos de agir" que encerram uma série de princípios e componentes técnicos. Aplica-se o termo "apoio" quando a tecnologia é utilizada para compensar uma limitação funcional e/ou facilitar um modo de vida independente.

O conceito de tecnologia de apoio deve ser sempre considerado em conjunto com o conceito de desenho universal que propõe que os espaços sejam projetados de forma a atender à população, considerando as variações de idade, sexo, tamanho, peso, habilidades ou limitações das pessoas. Estão envolvidos com a Tecnologia de Apoio os utilizadores finais e outros agentes. São considerados utilizadores finais pessoas com deficiências, com incapacidades transitórias e os idosos, familiares, cuidadores pessoais e outros agentes - profissionais de reabilitação, prestadores de serviços de TA (TA como instrumento para a atividade profissional) fabricantes, fornecedores, consultores de TA.

Ajudas Técnicas, segundo o Ministério da Saúde são os elementos que permitem compensar uma ou mais limitações funcionais motoras, sensoriais ou mentais com o objetivo de permitir-lhe superar as barreiras da comunicação e da mobilidade e possibilitar sua plena inclusão social.

Os conceitos aparentemente se justapõem buscando otimizar a relação homem- ambiente, o que implica também garantir conforto e gasto mínimo de energia, porém, faz-se necessário uma breve problematização dos mesmos com fins teóricos e práticos.

O conceito de Tecnologia Assistiva tem como eixo centralizador a relação indivíduo e tecnologia, onde a segunda pretende aumentar, manter ou melhorar as habilidades da pessoa com limitações funcionais, em uma relação direta e circunscrita a esta dualidade.

Já o conceito de Tecnologia de Assistência, apesar de sua definição estrita referir-se também à funcionalidade do indivíduo, está concebido numa abordagem ampliada de saúde proposta pela Classificação Internacional de Funcionalidade, Incapacidade e Saúde que "engloba todos os aspectos da saúde humana e alguns componentes relevantes para a saúde relacionados ao bem-estar e os descreve em termos de domínios da saúde e domínios relacionados à saúde" (OMS, 2003, p. 18).

Nesse contexto, a Tecnologia de Assistência está circunscrita aos domínios relacionados à saúde, onde a sua existência, acesso, custo, sentidos pessoais e culturais estão inter-relacionados aos componentes da funcionalidade e incapacidade (corpo, atividade e participação) e aos componentes dos fatores contextuais (fatores ambientais e pessoais), e, portanto, trata-se de uma compreensão de recursos tecnológicos que compõe uma concepção de saúde e funcionalidade de pessoas em grupos sociais e na apenas de uma resposta para incapacidades pessoais.

A concepção de Tecnologia de Apoio também contribui com a ampliação do conceito de "Tecnologia" para além dos objetos/equipamentos de auxilio na independência pessoal, e da relação direta com o usuário, situando-a em relação aos fatores humanos e socioeconômicos. Localizaa no âmbito dos contextos organizacionais, no de tecnologias que ajudem a ultrapassar as limitações funcionais dos seres humanos num contexto social, com propostas organizacionais e educativas da comunidade como um todo. Assim, a questão dos recursos tecnológicos deixa de ser uma questão específica da pessoa com incapacidade e pessoas próximas (familiares, terapeutas) e passa a situarse no contexto ampliado da sociedade, envolvendo Legislação/Economia, Normalização/Qualidade, Recursos de informação, produtores, vendedores, prestadores de serviços, organização de sistemas públicos de educação, saúde, transporte etc.

Em relação à definição do Ministério da Saúde de Ajudas Técnicas os recursos tecnológicos são problematizados a partir da inclusão, onde os equipamentos devem facilitar a participação social. Trata-se de uma definição ampla, com caráter mais político do que com uma preocupação com aspectos técnicos ou classificatórios.

Finalmente, destaca-se aqui, que as diferentes definições de recursos tecnológicos estão sendo concebidas sob a influência política das organizações de pessoas com deficiência, que teve como grande impulsionador o Ano Internacional da Pessoa com 
Deficiência (1981) e o Programa Mundial de Ação relativo às pessoas com Deficiência (ONU, 1992), quando enfatizaram os direitos das pessoas com deficiência às mesmas oportunidades que os outros cidadãos, bem como a uma partilha igual nas melhorias de condições de vida resultantes do desenvolvimento econômico e social.

\section{Aspectos relativos ao uso de recursos tecnológicos: breve revisão da literatura}

Muitas pesquisas foram realizadas nos Estados Unidos, no sentido de entender como e por que os usuários recursos tecnológicos decidem aceitar ou rejeitar o equipamento. Zhao e Phillips (1993) analisam e organizam esses estudos segundo: 1. características pessoais dos usuários e aceitação da tecnologia; 2. características do aparelho que os consumidores preferem e 3 . pesquisas de utilização do equipamento.

Propõem ainda outra pesquisa, bastante complexa, procurando descrever padrões gerais de uso e abandono de equipamento nos Estados Unidos.

A população alvo foi uma amostra de todo o país, de usuários de recursos tecnológicos - adultos com mais 18 de anos com uma variedade de incapacidades e deveriam ter experiência com pelo menos um aparelho. Foram 227 adultos com deficiência física que responderam à pesquisa. Relataram suas experiências com um total de 1732 aparelhos diferentes e 50\% foram abandonados. Outra meta principal neste estudo era identificar fatores associados com o abandono da tecnologia assistiva. E quatro fatores foram significativamente relacionados ao resultado de abandono, a saber: 1 . falta de consideração pela opinião do usuário; 2 . procura de aparelho fácil; 3 . desempenho ruim do aparelho; 4. mudança na necessidade ou prioridade do usuário.

De certa forma, este estudo proporciona uma ampla visão dos fatores importantes no uso bem-sucedido ou não dos recursos tecnológicos.

Com relação ao Brasil, essa trajetória também precisa ser feita. Mello (1998) destaca que apesar do reconhecimento dos benefícios trazidos pelo o uso dos equipamentos na qualidade de vida das pessoas com deficiência, a utilização deles no Brasil não prodigaliza. $\mathrm{E}$ aponta como principais fatores que contribuem para essa realidade o desconhecimento por parte dos técnicos e dos usuários da existência destes recursos tecnológicos, além do desconhecimento técnico por parte dos profissionais de reabilitação.

Quantos aos primeiros elementos para uma análise criteriosa sobre os alcances e limites da tecnologia aplicada, encontram-se nas reproduções das "falas" de usuários de um determinado Centro de Reabilitação em São Paulo, citadas na dissertação de mestrado de Nallin (1994). Nesse trabalho, a autora se propôs

estudar a reabilitação de pessoas portadoras de deficiência numa dada instituição, buscando resgatar os aspectos político-institucionais dessa prática, que são tradicionalmente, relegados pelo predomínio de uma visão assistencialista.

Faz a análise dos discursos de profissionais e clientes que, em última instância, são os suportes dessas práticas. Do material, pinçamos alguns trechos, preciosas referências ilustrativas nesse artigo, onde a autora apresenta a fala de uma paciente que demonstra o descompasso entre a demanda da pessoa e da instituição, que não o acompanha em seu processo interno:

Eu não vou comer com essa colher, porque eu nunca comi com colher torta. Isso é horrível, é feio. Eu jamais vou usar uma coisa assim. Então, eu prefiro não comer sozinha, que me dêem na boca (NALLIN, 1994, p. 131).

Prossegue a autora com o discurso da terapeuta ocupacional quando explica que as adaptações são individuais.

Uma adaptação básica é uma colher entortada aqui (mostra). Agora, eu não pego uma colher entortada e ponho na mão de todo mundo. Eu pego uma colher reta e eu vou entortar a colher na hora em que ele estiver comendo. Percebe? Então, eu vou entortar, moldando pra necessidade específica dele (NALLIN, 1994, p. 131).

Segundo Nallin (1994), a terapeuta passa a idéia de que a individualidade decorre, exclusivamente, das características anatômicas e funcionais, não fazendo menção à escolha, à opção pessoal.

A questão fica simplificada quando se considera que as adaptações são diferentes porque as pessoas realizam os movimentos diferentemente. Ainda nesse discurso, aparece a idéia de que o paciente, que é a verdadeira fonte de informações, não é quem informa. Comumente, as pessoas se dirigem aos acompanhantes dos deficientes para obter dados sobre eles, o que acentua o descompasso entre a demanda do paciente e o que a instituição lhe oferece.

Finalmente, o comentário da psicóloga da instituição sobre um paciente: Ele não fazia nada com a prótese. Ele continuava comendo com os pés (...) se trocava com os pés, uma loucura! (NALLIN, 1994, p.80).

Em sua dissertação de mestrado, Almeida (1993) procura compreender como a reabilitação impacta a vida cotidiana das pessoas com deficiência já desligadas dos serviços. A 
autora apresenta estudos de caso, dos quais selecionamos trechos significativos para o presente trabalho:

... eu recebi alta, ... eu saí de lá usando o aparelho com muletas. E eu sempre queria colocar o andador, mas eles falavam que não ia resolver nada o andador. ... já faz um ano que eu peguei o andador ... e, não aprendi lá, não. ... O meu aparelho eu uso da $1 \mathrm{~h} 00$ até as $5 \mathrm{~h} 00$ com o andador, pra dar aula à tarde. Eu queria o andador, mais pra poder facilitar as coisas pra mim, mas não porque gosto (p. 48-9).

A autora afirma que o uso do andador foi dotado de sentido nas situações vividas cotidianamente; no entanto, a indicação, orientação e treino não tinham feito parte do seu processo de reabilitação.

Em outro depoimento:

... Assim, do jeito que eu estou, sentado, eu não me sinto uma pessoa deficiente física. Na cadeira de rodas, eu não me sinto assim. Mas naquela órtese, eu me sinto, eu fico todo torto e não consigo fazer nada (p. 63).

Nesse caso, a autora apresenta razões para a rejeição do aparelho: a ineficácia do equipamento, que gera insegurança, e seu uso, que denuncia as impossibilidades impostas à auto-estima.

Em sua tese de doutorado, Mello (1998) avalia a necessidade e o uso de equipamentos de auto-ajuda e adaptações ambientais por idosos com comprometimento funcional, com a finalidade de aumentar a independência na comunidade.

O trabalho examina os equipamentos ou adaptações que estão sendo utilizados por essa população, determina o nível de satisfação dos usuários e identifica o profissional responsável pela prescrição dos aparelhos, pelo treino e segmento do uso, além das fontes financiadoras desses equipamentos. Apresenta como resultado um dado relevante para o nosso estudo: dos 62 casos que possuem algum tipo de equipamento de auto-ajuda: $29 \%$ - estão satisfeitos; $6,5 \%$ - pouco satisfeitos; $16,1 \%$ - indiferentes; $12,9 \%$ - pouco insatisfeitos; $14,5 \%$ - muito insatisfeitos, mas continuam usando; $16,1 \%$ - insatisfeitos a ponto de terem abandonado o equipamento; $4,8 \%$ - recusaram-se a responder.

$\mathrm{Na}$ conclusão, a autora afirma que os idosos dependentes tinham baixo poder aquisitivo, e baixo índice no uso dos equipamentos de auto-ajuda e de adaptações ambientais que poderiam e lhes proporcionar maior autonomia e independência em seus domicílios. No entanto, não existe financiamento efetivo por parte do sistema público de saúde de equipamentos de auto-ajuda, apesar de estar previsto em leis federais, estaduais e municipais. A autora recomenda o aprimoramento do sistema de concessão de órteses e próteses do Governo do Estado de São Paulo, e a formação de equipes interprofissionais, treinadas para a avaliação das necessidades, buscando prevenir o abandono dos equipamentos prescritos. Surge também a elaboração de instrumentos que permitam medir a efetividade do uso e a melhora da qualidade de vida dos usuários, além da implantação de sistemas de segmento de uso de equipamentos de auto-ajuda pelos usuários idosos.

Também em tese de doutorado, Rocha (1999) traz questões importantes para essas reflexões. Analisa criticamente o campo da reabilitação de pessoas portadoras de deficiência, e aponta quatro concepções de reabilitação - ortopédica, fisiátrica, baseada na comunidade e ecológica, ou de vida independente.

Essas concepções são postas em prática de formas diferente por serviços filantrópicos e particulares e pelo campo da saúde pública, e dentro delas, a autora destaca a diversidade da aplicação da tecnologia assistiva, estabelecendo os nexos históricos e epistemológicos entre os conceitos, as propostas e as práticas desenvolvidas.

A tecnologia assistiva da concepção ortopédica é aplicada para corrigir, dar apoio (órteses) ou substituir membros ou partes do corpo (próteses) (p. 84).

Na concepção fisiátrica, (...) os recursos da tecnologia assistiva são utilizados para compensar e/ou complementar as funções alteradas, ou quando o máximo de recuperação das mesmas não foi atingido pelas intervenções tradicionais de reabilitação como os exercícios (p. 85).

Na reabilitação baseada na comunidade (RBC), (...) os recursos, as técnicas reabilitadoras, os técnicos e as tecnologias são adaptados às alternativas existentes no ambiente, executadas até por leigos, feitas de material acessível, de confecção rústica e/ou caseira. (...) A tecnologia assistiva nessa abordagem é um meio, não sendo muito importante sua qualidade, do ponto de vista dos aspectos específicos do corpo do indivíduo, nem tampouco a correção em si (p. 86).

$\mathrm{Na}$ abordagem de vida independente, (...) a tecnologia busca a equiparação de oportunidades para todos, por meio de intervenções sobre a pessoa; o meio ambiente, os processos de tecnologia assistiva e de sensibilidade ambiental se justapõem (p. 89).

A partir desses dados, Rocha (1999) aponta uma realidade bastante discrepante: de um lado, propostas de Centros de Vida Independente (CVI), com alta tecnologia e, como nos EUA, no outro extremo, o RBC, com tecnologia simplificada, em países empobrecidos. Segundo a autora, tais equipamentos - que nem sempre são necessários ou eficazes para a pessoa com deficiência, provocam as vezes novas incapacidades.

Também conclui que os equipamentos de ajuda não são a resposta para a totalidade dos problemas enfrentados pelas 
pessoas com incapacidades, mesmo em países ricos, e inclusive os organismos internacionais têm dúvida, como vimos.

Em sua dissertação de mestrado, Wolff (2001), a partir de sua atividade clínica fonoaudiológica, "propõe-se estudar as possibilidades de crianças com transtornos invasivos do desenvolvimento (TID) terem contato com outros através de comunicação alternativa”. Constatou que as crianças perceberam que o uso da prancha e a escolha dos símbolos geram um efeito desejado, e passaram a utilizá-la e a ampliar sua linguagem. No entanto, menciona que muitos autores afirmam que o uso da comunicação alternativa deixa uma lacuna - treinam-se as crianças para utilizar os símbolos decorando-os, mas não se consegue que elas usem esses símbolos fora do contexto terapêutico.

Wolff (2001) acredita que as dificuldades não serão superadas apenas aprimorando a tecnologia. Diz que há momentos em que a prancha não é necessária, e conclui que não se substitui o que já funciona: a prancha será utilizada quando a comunicação não oral não for suficiente.

Em suas considerações finais, pondera que não conseguiu descobrir por que esses símbolos, com as interpretações do terapeuta, favoreceram a interlocução. Levanta hipóteses como a de dificuldade ou a não aceitação da fala, a dificuldade verbal neurofisiológica ou um misto dessas duas alternativas, ou ainda algo que não está ao nosso alcance imaginar. Deixa a questão aberta e aponta a necessidade de outros tipos de investigação.

Para colaborar com esse debate sobre a relação entre tecnologia e pessoas com deficiência, Castiglioni (2003) em seu doutorado faz uma análise crítica da tecnologia assistiva. Parte do suposto que a compreensão da função dela envolve várias dimensões: mecânica-instrumental; ético-política e afetiva-subjetiva. Ou seja, a discussão sobre tecnologia que envolve habilidades mecânicas e funcionais, passa a envolver também necessidades e sofrimentos. Para avaliar a tecnologia assistiva e entender o que faz com que as pessoas usem ou não todo o aparato tecnológico, é preciso ir além dos critérios exclusivamente quantitativos. Não se pode negligenciar o aspecto humano, e sim considerar a pessoa real com suas carências e necessidades mais elevadas, assim como não se pode deificar a tecnologia como um avanço, um bem em si, e, assim, estranhar os que a recusam.

Para ir além da análise da acessibilidade e os entraves colocados pelos serviços públicos; a permanência no uso também precisa ser contemplada na discussão. $\mathrm{O}$ acesso e os entraves econômicos são questões de justiça social e desigualdade. A permanência envolve também o sentido que a tecnologia assistiva tem para as pessoas.

\section{Concluindo: o recurso tecnológico como instrumento de inclusão social?}

As pessoas com deficiências ou incapacidades vivem, ainda, via de regra, situações de segregação e de exclusão social. Desenhar a utopia da inclusão é antes de tudo analisar a os processos de exclusão. As definições de Recursos Tecnológicos (Tecnologia Assistiva, de Assistência, de Apoio e de Ajudas) apontam, por diferentes caminhos para o paradigma da inclusão social da pessoa com deficiência. Essas abordagens inseridas em contextos diversos podem atingir ou não a meta proposta, ou seja, apenas o acesso aos equipamentos não garante a efetivação da finalidade proposta. Faz-se necessário problematizar a relação entre recursos tecnológicos, independência e autonomia e a dialética inclusão e exclusão social.

A partir de Sawaia (1999) podemos problematizar o conceito de Inclusão. Para a autora inclusão e exclusão implicam em uma relação dialética, onde todos estão inseridos de algum modo, ainda que à margem do "corpo social" e pelos mecanismos da exclusão. A sociedade exclui para incluir. E não se trata de uma falha no sistema, mas de uma seqüência regular e necessária para sua perpetuação nos moldes atuais. Assim sendo, a exclusão e inclusão não constituem categorias em si, pois formam um par indissociável que se constitui na própria relação.

A idéia de inclusão pela exclusão ajuda a definir uma estratégia de domínio e exploração, um modo de vida que compromete a capacidade de ser cidadão, em que os direitos estão sob risco permanente de extinção. Trata-se de uma distribuição desigual dos direitos e dos bens materiais. E as formas dessas ocorrências nesta sociedade são múltiplas: com os idosos, com as minorias étnicas, com os desempregados há muito tempo, com as pessoas com deficiências ou incapacidades. Essas pessoas são vistas como estando aquém da humanidade.

Nesse sentido, Sawaia (1999) elege o sofrimento como a principal categoria de análise psicossocial do processo de exclusão/ inclusão, e qualifica-o como ético-político. Para a autora, a exclusão vista como sofrimento de diferentes qualidades recupera, por assim dizer, o indivíduo perdido nas análises econômicas e políticas, sem deixar de lado, entretanto, o coletivo. Ou seja, o foco de atenção direcionase para o indivíduo que nem se quer pode desenvolver seu potencial humano, em função da natureza restrita a que está submetido.

Sawaia (1999) afirma ainda que o sofrimento ético político mutila a vida de diferentes formas, representando com exatidão as questões sociais dominantes vividas cotidianamente em cada momento histórico. 
Deparamo-nos aqui com a problemática de como lidar, não só teoricamente, mas também no cotidiano, com esse fenômeno aplicado à problemática da pessoa com deficiência. Para tanto, se faz necessário à presença de uma postura ética de rompimento com o descompromisso político frente ao sofrimento do outro, contemplando os aspectos subjetivos da constituição das desigualdades para além de ações legalistas ou politicamente corretas.

Enfrentar os mecanismos de exclusão/ inclusão, implica entendê-los em suas constituições particulares, dirigidas a problemas específicos. Intervir primeiramente na explicitação de seu significado através de um debate entre os envolvidos com a questão e a partir de então, construir novos sentidos na relação de todos com a problemática em pauta.

Essas reflexões suscitam o questionamento ético- político dos recursos tecnológicos orientando o olhar à qualidade de inclusão que promovem.

Os recursos tecnológicos não são em si nem facilitadores, nem dificultadores de processos de inclusão social, de satisfação e realização pessoal e de grupos sociais, tão pouco promotores de independência e autonomia. Considera-se aqui, que a utilização dos mesmos deve estar contextualizada em processos de construção de histórias de vida particulares, inseridas em processos de exercício pleno da cidadania e de felicidade dos seus usuários. Para tanto, há de se reafirmar a necessidade de abordagens que considerem os aspectos que estão presentes nas histórias de vidas das pessoas com deficiência, os sentidos que os equipamentos ou a falta de acesso a eles tem para essas pessoas, os sentidos sociais, educacionais e políticos presentes.

ROCHA, E. F.; CASTIGLIONI, M. C. Reflections on technological resources: aids techniques, assistive technology, technology of assistance and technology of support. Rev. Ter. Ocup. Univ. São Paulo, v. 16 , n. 3, p. 97-104, set./dez., 2005.

\begin{abstract}
Specialized technological resources, helping devices, are being produced more frequently in the last few years and some terms which synthesize these productions have been created. In Brazil, many terminologies are being used to define what technological resources mean such as: Assistive Technology (USA), Technology of Assistance (CIF/OMS) and Technology of Support (European Committee /EUSTAT) and Aids Techniques (Health Department). Due to those different conceptions around this knowledge field, there are many different categorizations as well as unique ways of understanding this theme. Strategies and procedures that go are also distinct and posterior to their related comprehension. The present work intends to discuss the technological resources beyond the practical aspects in its everyday usage. We assume that the TA (Assistive Technology, of Support, of Assistance or Aids Techniques) is a multidimensional phenomenon, which involves mechanical, biomechanical, ergonomic, functional, kinetic, ethical, aesthetic, politicial, affective, and subjective aspects, and must be analyzed like that. It also presents the concepts implied in it, in accordance with the different conceptions of Rehabilitation. Finally, we analyze the direction and purpose of these resources in the process of social inclusion of handicapped people.
\end{abstract}

KEY WORDS: Biomedical technology/trends. Technology/trends. Self-help devices. Disabled persons/ rehabilitation. Rehabilitation.

\title{
REFERÊNCIAS
}

CASTIGLIONI, M. C. Entre o exílio e a libertação: uma analise psicossocial da tecnologia assistiva. 2003. Tese (doutorado) - Pontifícia Universidade Católica de São Paulo, São Paulo, 2003.

EUSTAT. Educação em tecnologias de apoio para utilizadores finais: linhas de orientação para formadores. Comissão Européia,1999. Disponível em: http://www.siva.it/research/eustat/ estsgupt.html. Acesso em: 02 maio 2004.

MELLO, M. Tecnologia assistiva. In: GREVE, J. M. D.; AMATUZZI, M. M. Medicina de reabilitação aplicada à ortopedia e traumatologia. São Paulo: Manole, 1997.

MELLO, M. A necessidade de equipamentos de auto-ajuda e adaptações ambientais de pessoas idosas dependentes vivendo 
na comunidade. 1998. Tese (Doutorado) - Universidade Federal de São Paulo - UNIFESP, São Paulo, 1998.

NALLIN, A. Reabilitação em instituição: suas razões e procedimentos. Análise de representação do discurso. Brasília: CORDE - Coordenadoria Nacional para integração da pessoa portadora de deficiência, 1994.

OMS - ORGANIZAÇÃO MUNDIAL DA SAÚDE. CIF: Classificação Internacional de Funcionalidade, Incapacidade e Saúde [Centro Colaborador da Organização Mundial de Saúde para a Família de Classificações Internacionais, org.; coordenação da tradução Cássia Maria Buchalla]. São Paulo: Editora da Universidade de São Paulo, 2003.

ONU - ORGANIZAÇÃO DAS NAÇÕES UNIDAS [1982]. Programa de ação mundial para as pessoas com deficiência. São Paulo, CEDIPOD - Centro de Documentação e In-

Recebido para publicação: 10/09/2005

Aceito para publicação: 30/09/2005 formação do Portador de Deficiência, 1992.

ROCHA, E. F. Do corpo orgânico ao corpo relacional: uma proposta de deslocamento dos fundamentos e práticas de reabilitação da deficiência. 1999. 237f. Tese (doutorado) Instituto de Psicologia da Universidade de São Paulo, São Paulo, 1999.

SAWAIA, B. As artimanhas da exclusão: análise psicossocial e ética da desigualdade social. Petrópolis: Vozes, 1999.

WOLFF, L. M. G. Comunicação suplementar e/ou alternativa nos transtornos invasivs do desenvolvimento. Dissertação (mestrado) - Pontifícia Universidade Católica de São Paulo, São Paulo, 2001.

ZHAO, H.; PHILLIPS, B. Predictors of assistive technology abandonment. Assist. Technol., v. 5, n.1, p. 35-45, 1993. 